\title{
Filtration suppresses laser induced nucleation of glycine in aqueous solutions
}

\author{
Nadeem Javid ${ }^{1,2 \ddagger}$, Thomas Kendall ${ }^{1,3 \ddagger}$, Iain S. Burns ${ }^{1}$ and Jan Sefcik ${ }^{1,3 *}$ \\ ${ }^{1}$ Department of Chemical and Process Engineering, University of Strathclyde, 75 Montrose \\ Street, Glasgow, UK, ${ }^{2}$ Strathclyde Institute of Pharmacy and Biomedical Sciences, University of \\ Strathclyde, 99 George Street, Glasgow, UK ${ }^{3}$ CMAC, Technology and Innovation Centre, \\ University of Strathclyde, 99 George Street, Glasgow, UK.
}

Keywords: Non-Photochemical Laser Induced Nucleation, Crystallisation, Polymorphism, Glycine, Nanofiltration, Nucleation Kinetics

Abstract: We demonstrate that nanofiltration of aqueous glycine solutions has a pronounced effect on laser-induced nucleation. Two nucleation regimes were observed in non-filtered, irradiated solutions under isothermal conditions: a rapid initial regime associated with laser induced nucleation, and a second much slower spontaneous nucleation regime. Filtration of the solutions prior to irradiation greatly suppressed the rapid regime, while the slow regime was similar regardless of filtration or irradiation, for all supersaturations studied. A clear effect of filtration on crystal polymorphism was also observed. Non-filtered irradiated solutions at a lower supersaturation almost exclusively yielded the $\alpha$-polymorph, while at higher supersaturations there was significant presence $(\sim 40 \%)$ of the $\gamma$-polymorph. On the other hand, filtered solutions 
almost exclusively yielded the $\alpha$-polymorph of glycine at all supersaturations studied. These surprising results challenge some established ideas about laser induced nucleation, showing that previously reported laser induced nucleation phenomena in glycine aqueous solutions can be effectively suppressed by filtration, so that the underlying mechanism is unlikely to be based on molecular scale interactions involving just the solute and the solvent alone. Instead, laser induced nucleation in this system appears to be related to either colloidal scale solution clusters or foreign solid or molecular impurities that can be removed by nanofiltration.

Introduction: Control of polymorphism and corresponding nucleation kinetics is crucial to the development of robust crystallisation processes in pharmaceutical and chemical manufacturing. Nucleation behaviour impacts directly on the solid form and indirectly on particle shape and particle size distribution, which are among critical quality attributes of particulate products influencing economical and therapeutic performance ${ }^{1,2,3}$ of pharmaceuticals. Various methods are available to induce crystallization from solutions. Traditional approaches such as cooling, evaporation, reaction or anti-solvent addition have been more recently augmented by using external fields, including ultrasound, shear or electric fields. ${ }^{4,5,6,7,8}$ New techniques that can provide improved control of nucleation are of great interest for development of reliable, robust and reproducible crystallisation processes in the pharmaceutical, fine chemical and materials manufacturing sectors.

It has been previously demonstrated that short pulses of high power laser light can be used as an external field to induce nucleation of various compounds from solutions without any apparent photochemical effects. ${ }^{9}$ Non-photochemical laser induced nucleation is a term used in the literature to refer to nucleation induced by laser light, continuous or pulsed, where the solution is 
nominally non-absorbing at the laser wavelength used and thus no significant photochemical effects are expected to occur in the solution. Laser induced nucleation offers an attractive way of achieving well-controlled primary nucleation, with spatio-temporal localisation of the nucleation event. ${ }^{\text {Error! Bookmark not defined.10 }}$ This method has been shown to work across a wide range of compounds, including inorganic salts, amino acids, proteins, and small organic molecules such as carbamazepine. ${ }^{11}$ More recently, it has been shown that under certain conditions, laser induced nucleation allows for some control of polymorphism in supersaturated glycine solutions based on the polarization of laser light ${ }^{12,13,14}$ and a similar result has been demonstrated in continuouswave optical trapping experiments. ${ }^{15}$

The mechanism by which non-photochemical laser induced nucleation works in aqueous solutions is unknown, but different theories have been proposed, involving interactions of the laser with individual solute molecules ${ }^{9}$, with subcritical nuclei, ${ }^{16}$ or with suspended nanoparticles ${ }^{17}$ or involving cavitation which in turn induces nucleation ${ }^{18}$ as discussed further below.

A molecular level theory was proposed by Garetz et al ${ }^{12}$ where observations about polymorphic control, directional influence of crystal formation and probability of nucleation were linked to the polarization of light, which led to the suggestion of the Optical Kerr Effect being involved in a nucleation mechanism. In the Optical Kerr Effect, the electric field induces a dipole in molecules or clusters of molecules, causing the molecules to align and thus reducing the entropic contribution in activation free energy for nucleation. However, Knott et al ${ }^{19}$ have shown that the energy reduction by torque alignment would not be sufficient to account for the observed reductions in nucleation time reported. Another proposed mechanism is isotropic polarizability of sub-critical nuclei which is based on the classical nucleation theory. ${ }^{20}$ Sub- 
critical nuclei in solution experience polarization from the electric field of the laser pulses that lowers the free energy barrier for nucleation.

Cavitation was directly observed to lead to nucleation under irradiation of supersaturated solutions (containing substances absorbing laser light to facilitate creation of cavitation bubbles) by nanosecond laser pulses. ${ }^{18}$ Nakamura et $a l^{21}$ have shown that highly focussed femtosecond laser pulse creates similar effects as ultrasound induced cavitation, where the solution undergoes localised heating and vaporisation resulting in the formation of a bubble which rapidly expands to a critical size. In either case, the bubble surface either acts as heterogeneous nucleation location or, upon collapsing generates a high localised concentration gradient, thus inducing crystallisation. Alexander and co-workers studied laser induced nucleation of carbon dioxide bubbles and proposed that heating of suspended nanoparticles by the laser may be responsible for formation of bubbles. ${ }^{17}$

Another way of looking at potential mechanisms of laser induced nucleation would be to divide proposed explanations into those based on: a) interaction of the laser directly with solute molecules or their molecular scale clusters in the solution, b) interaction of the laser with molecular impurities (either photochemical or non-photochemical) and c) interaction of the laser with colloidal scale particles or larger scale impurities (e.g., dust) present in nominally clear solutions. Potential explanations could then be tested by removing or adding molecular or particulate impurities and investigating their effects on laser induced nucleation phenomena. In particular, adsorbents or filters could be used to try to purify or isolate soluble or suspended components of solutions to be subject to laser irradiation. For example, we proposed previously that large mesoscale clusters in amino acid aqueous solutions may be involved in preferred nucleation pathways in these systems. Nanofiltration of these solutions can be used to decrease 
the number and size of these clusters significantly while they can be reformed in solution after extended periods of time. ${ }^{22,23}$ Similarly, if external nanoparticulate impurities were involved, nanofiltration would serve to remove them.

Nanofiltration with 0.2 micron cellulose acetate filters was used previously in laser induced nucleation studies on potassium chloride ${ }^{16}$ and although no quantitative comparison was made with non-filtered solutions it was stated that "solutions that were not filtered ... are significantly more labile to nucleation" (i.e., higher proportion of irradiated vials nucleated within a given time interval). However, it was noted that filtered $\mathrm{KCl}$ solutions, even when using high purity samples and clean preparation, still showed clear enhancement of nucleation above a certain peak power density threshold. Laser induced nucleation of lysozyme and trypsin was investigated $^{24}$ using nanofiltered solutions, which were mixed with presumably unfiltered precipitant solutions before being irradiated, although no comparison with completely unfiltered solutions was made. Another case where nanofiltration and purification was investigated is nucleation of carbon dioxide bubbles. Threshold pulse energy to induce bubble nucleation was found to be similar for solutions prepared using either tap or ultrapure water. ${ }^{19}$ Aqueous sucrose solutions supersaturated with carbon dioxide were found to be significantly less prone to bubble nucleation when filtered and purified, while again no large differences in threshold peak power density were seen between filtered and unfiltered solutions. ${ }^{17}$ We are not aware of any other published studies where effects of nanofiltration on laser induced nucleation were investigated.

In order to better understand laser induced nucleation phenomena and to improve our ability to control these processes to our advantage, we have investigated effect of nanofiltration on laser induced nucleation of glycine in aqueous solutions to determine whether colloidal scale structures or particulate impurities are involved in the mechanism of laser induced nucleation. 
We have also quantitatively studied kinetics of laser induced nucleation and for the first time estimated nucleation rate constants of both laser induced and spontaneous nucleation in the same solution. We irradiated filtered and non-filtered solutions with linearly-polarized nanosecond pulsed laser light $(\lambda=1064 \mathrm{~nm})$ under isothermal conditions at various supersaturations. A cumulative distribution function of induction times for non-filtered irradiated solutions revealed the presence of two nucleation regimes, a rapid initial regime associated with laser induced nucleation, and a second much slower spontaneous nucleation regime. Filtration of the solutions prior to irradiation greatly suppressed the rapid regime while the second, slow nucleation regime was observed for all supersaturations studied; indicating that the underlying mechanism of laser induced nucleation is unlikely to be based solely on molecular scale interactions involving just the solute and the solvent alone.

Materials and Methods:

\section{$\underline{\text { Solution Preparation and Laser Irradiation }}$}

Glycine (Sigma Aldrich, electrophoresis $\geq 99 \%$ ) was used without further purification and deionised water with conductivity lower than $15 \mu \mathrm{S} / \mathrm{m}$ was obtained from an in-house dispenser (Millipore $1.1 \mathrm{M} \Omega \mathrm{cm}$ ). Solutions were prepared in an incubator at $333 \mathrm{~K}$ using a magnetic stirrer, at three glycine concentrations, $350 \mathrm{~g}$ of glycine $/ \mathrm{kg}$ of water, $375 \mathrm{~g}$ of glycine $/ \mathrm{kg}$ of water and $400 \mathrm{~g}$ of glycine/kg of water, with the corresponding relative supersaturations at $298 \mathrm{~K}$ ranging from 1.4 to 1.6 (based on glycine solubility $25 \mathrm{~g} / 100 \mathrm{~g}$ water ${ }^{25}$ ) at $298 \mathrm{~K}$. Small HPLC vials (Scientific Glass Laboratories, part number T101/V1, diameter 11mm, volume 2ml) with screw caps were cleaned with deionised water and kept in the incubator at $333 \mathrm{~K}$ along with syringes and sterile filters $\left(0.2 \mu \mathrm{m}\right.$ Polyethersulfone (PES), Millipore Express ${ }^{\circledR}$ PLUS Membrane Filters). When filters were used $10 \mathrm{ml}$ of solution was flushed through first and discarded to 
remove any trace chemicals on the filter membrane. The solutions were hot-filled in the HPLC vials (with or without filtration) at $333 \mathrm{~K}$ and then gradually cooled to $298 \mathrm{~K}$ over $3-4$ hours followed by holding for 20 hours at 298 K. Samples which crystallised within this time were removed before proceeding further. It has been suggested previously that length of holding time appeared not to influence the outcome of laser-induced nucleation of glycine ${ }^{26}$ but this time was used for practical reasons to keep holding time consistent for all samples. The vials were transferred to a temperature controlled water bath kept at $298 \mathrm{~K}$ and from there they were moved to a Peltier temperature controlled sample holder and exposed to 1 minute of irradiation by a pulsed Nd:YAG laser (Continuum Surelite II-10) with a wavelength of $1064 \mathrm{~nm}$ (pulse duration: $6 \mathrm{~ns}$; repetition rate: $10 \mathrm{~Hz}$; linear polarization). A $1 \mathrm{~mm}$ circular pin-hole was used to select the central portion of the Gaussian beam, resulting in spatial profile that was near to top-hat. It should be noted that the laser spatial profile will change considerably as it passes through the circular sample vial, which acts as a powerful cylindrical lens, just as it does in previous studies of laser induced nucleation. The desired optical power density was generated by passing the laser light through a home-built telescope before it reached the $1 \mathrm{~mm}$ pinhole. All the work reported here was done at a power density of $0.47 \mathrm{GW} / \mathrm{cm}^{2}$ (calculated based on a circular, top-hat spatial profile and a top-hat temporal profile).

The experimental design consisted of four sets of experiments: 1) Non-filtered, irradiated samples; 2) Non-filtered, non-irradiated samples as control experiments to differentiate the spontaneous nucleation from laser induced nucleation; 3) Filtered, irradiated samples to test the effects of filtration on laser induced nucleation; 4) Filtered, non-irradiated samples to test the effects of filtration on spontaneous nucleation. Three different solution concentrations were studied at isothermal conditions at temperature $298 \mathrm{~K}$. This results in a total of 12 sets of 
experimental conditions. Experiments were performed for each set of conditions on at least 50 samples (in total 760 samples) keeping in mind the stochastic nature of the nucleation process.

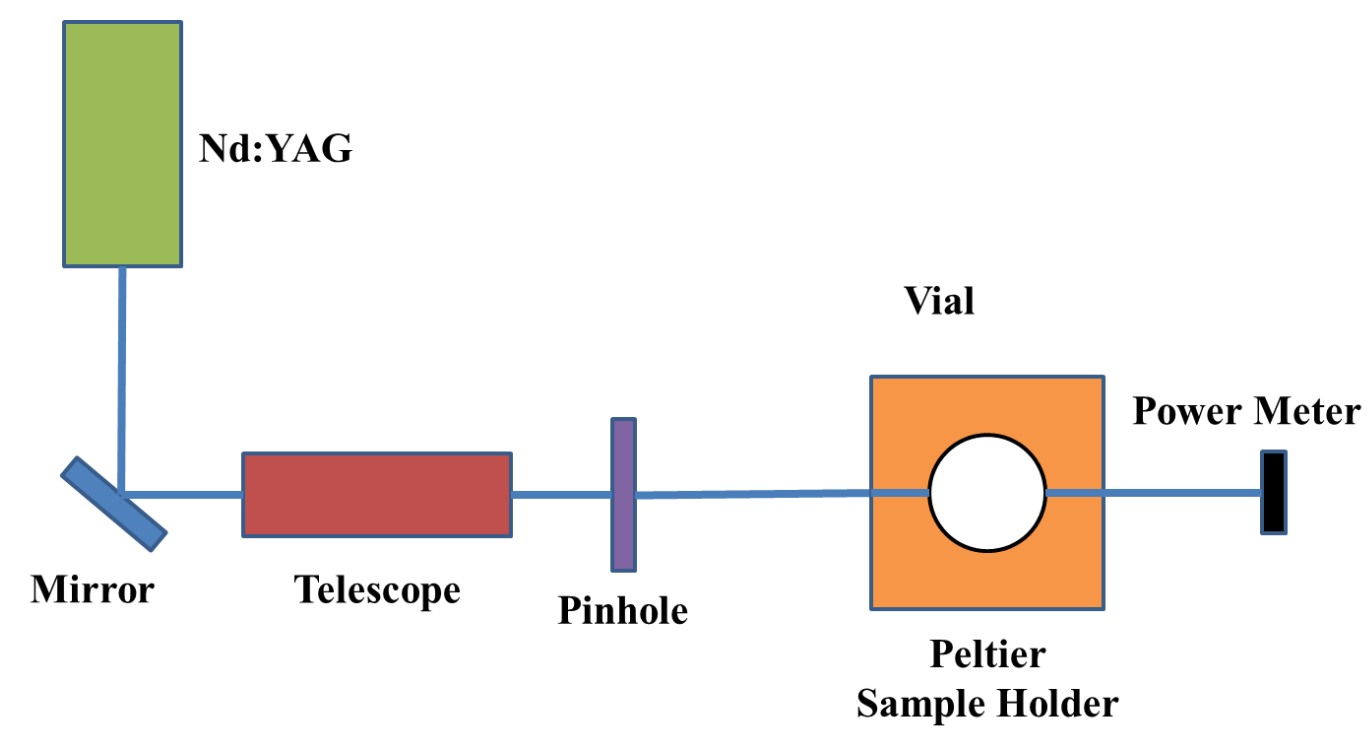

Figure 1: Schematic representation of laser irradiation set up

\section{Imaging and Data Processing}

All samples were placed in a temperature controlled bath immediately after irradiation and were then transferred to a vial rack in an incubator set at $298 \mathrm{~K}$. Images of all the vials were recorded every 2 minutes for four days using a webcam with time-lapse software. Induction times were obtained from the analysis of image files by filtering and using a threshold intensity to identify crystals and estimate their size. The induction time is defined here as the period of time between the laser irradiation of samples under isothermal conditions for given supersaturation and the detection of crystals at constant temperature (298 K). Detection of crystals was based on time when the size of a crystal reached $1 \mathrm{~mm}$.

The cumulative probability distribution function of the induction time can be determined from a large number of induction time measurements at given supersaturation and constant 
temperature as described by Jiang and ter $\operatorname{Horst}^{27}$. For $N$ individual samples in repeated experiments, the probability $P(t)$ that an induction time is between zero and time $t$ is defined as $P(t)=\frac{N^{+}(t)}{N} \quad$ Eq. 1

where $N^{+}(t)$ is the number of samples where crystals have been detected up to a given time $t$.

\section{Attenuated Total Reflection-Fourier Transform Infrared Spectroscopy (ATR-FTIR)}

Crystals formed were extracted from the vials for solid form characterization using an ATRFTIR spectrophotometer with a diamond cell (Clairet Scientific , MB 3000). For each spectrum, 16 scans were collected in absorption mode with a resolution of $4 \mathrm{~cm}^{-1}$ at room temperature and analyzed in the range of $800-1100 \mathrm{~cm}^{-1}$ for polymorph characterization. Reference spectra (air) were recorded under identical conditions.

\section{$\underline{\text { X-Ray Diffraction (XRD) }}$}

All X-ray diffraction measurements were performed using the Oxford Diffraction Xcalibur E instrument at $123 \mathrm{~K}$ with Mo radiation. Each cell is based on 15 frames of data, each frame corresponding to a 1 degree rotation in omega. Cells are based on approximately 25 to 90 reflections each. CrysalisPro software was used to generate the unit cells.

\section{Results and Discussions:}

\section{$\underline{\text { Effects of nanofiltration and laser irradiation on nucleation kinetics }}$}

Induction times (the time of appearance of a visible crystal after laser irradiation) for samples at different supersaturations were determined by analyzing the digital images and were used to calculate the cumulative probability distribution functions of induction time (Equation 1); the 
results are shown in Figure 2. The probability of crystals appearing within four days is relatively low in the absence of irradiation (for both filtered and non-filtered solutions), while it is much higher in non-filtered irradiated solutions (Table 1), increasing with increasing supersaturation as expected. This is consistent with results reported previously at comparable glycine concentrations and laser power densities ${ }^{26}$. A striking feature of these results is that the effect of laser irradiation on enhancing nucleation probability is strongly suppressed for filtered samples. This is a surprising result, which challenges some established ideas about laser induced nucleation phenomena previously reported in this system, as it strongly suggests that the underlying mechanism is unlikely to be based on molecular scale interactions involving just the solute and the solvent alone. The effect of irradiation and filtration on nucleation kinetics in glycine solutions at different supersaturations is compared and described in more detail below.

a) Non-filtered non-irradiated samples: The probability of nucleation is low in the absence of laser irradiation (Figure 2, empty squares): for example, the proportion of samples nucleated after 4000 minutes is around $10 \%$ for supersaturation 1.5. This provides a base case for comparison with irradiated samples.

b) Non-filtered irradiated samples: The probability of nucleation is far greater than for nonfiltered, non-irradiated samples. The cumulative probability of nucleation increases with increasing supersaturation (Figure 2, filled squares). Observations made here about the dependence of nucleation probability on concentration were also seen in the previous work by Sun et $a l .{ }^{13}$ and Clair et $a l .{ }^{26}$ The cumulative probability distribution functions for the nonfiltered, irradiated samples appear to show two distinct regimes of nucleation. The initial regime, characterized by a steep slope, is a fast nucleation regime, which is dominant during the first tens to hundreds of minutes after irradiation. The second regime, with a more gradual slope, is a slow 
nucleation regime with induction times extending to thousands of minutes. The kinetics of nucleation can be qualitatively seen to be increasing with increasing supersaturation as would be expected. It is also worth pointing out that the second slow regime for non-filtered irradiated samples seems to qualitatively resemble that for non-filtered non-irradiated samples. Further quantitative analysis of cumulative probability distribution functions of induction times will be presented in the nucleation kinetics section below.

c) Filtered irradiated samples: Surprisingly, the nanofiltration of glycine solutions resulted in a massive decrease in the probability of laser induced nucleation at all supersaturations. The nucleation probability over three days decreased by factors of approximately 7, 5 and 3 for supersaturations 1.4, 1.5 and 1.6, respectively, as compared to non-filtered irradiated samples (Figure 2, filled circles). Additionally, it is clear that the first fast nucleation regime is greatly suppressed in these filtered samples. The nucleation probability increases gradually with increasing supersaturation as observed in previous cases. As described in Materials and Methods section, the glycine solutions were hot filtered at $333 \mathrm{~K}$ with PES filters having pore size of 0.2 $\mu \mathrm{m}$. This procedure therefore cannot remove glycine molecules from solution but is expected to filter out particulates with size larger than filter pore size or remove molecular or particulate impurities that can be adsorbed on the filter membrane. The results therefore point to a mechanism for laser induced nucleation that is strongly dependent on the presence of impurities or particulates, of solute, solvent or external origin, that can be removed by filtration. 
A)

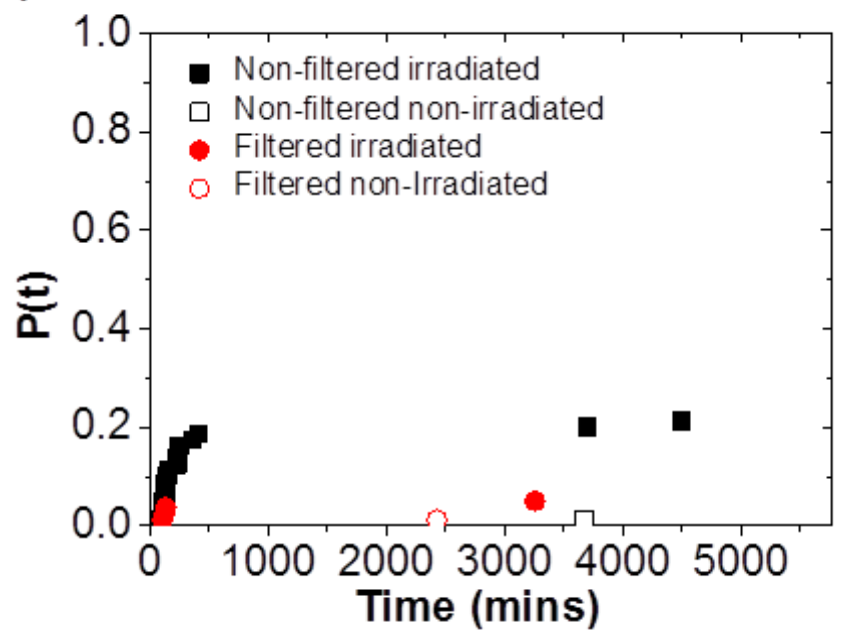

B)

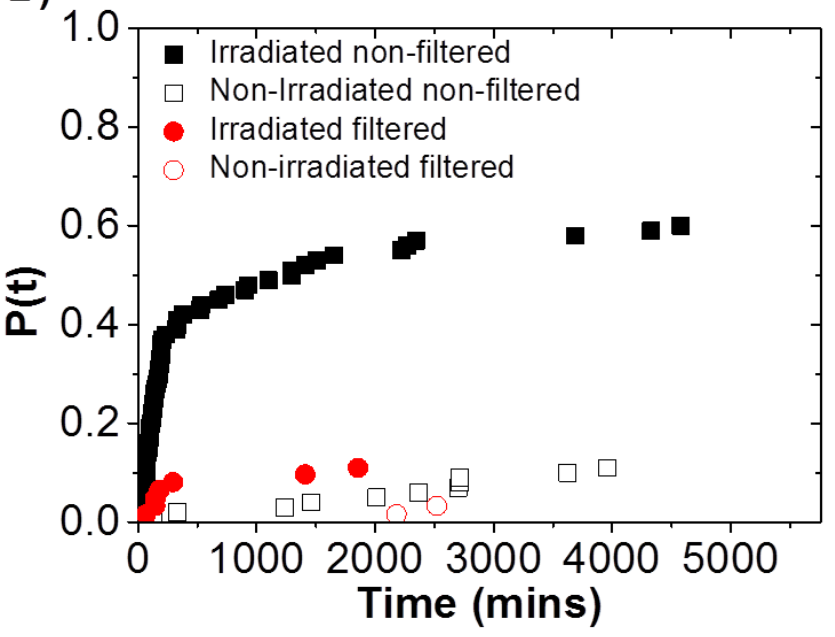




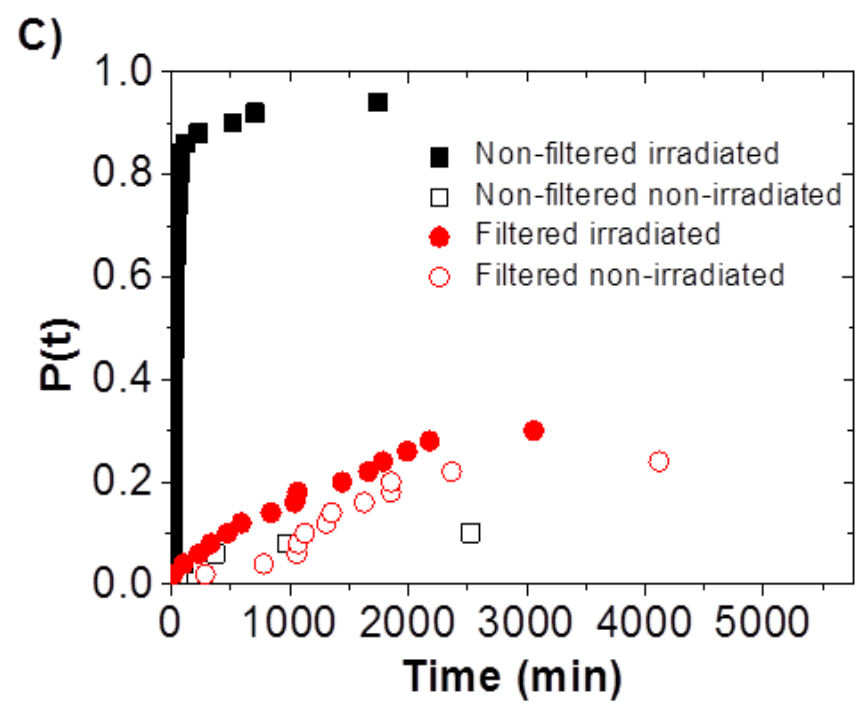

Figure 2: Cumulative probability distribution functions of induction times in supersaturated glycine aqueous solutions at three different supersaturations: A) 1.4, B) 1.5, C) 1.6, where filled symbols represent irradiated samples and hollow symbols represent non-irradiated samples, squares represent non-filtered samples and circles represent filtered samples.

d) Filtered non-irradiated samples: The probability of nucleation for filtered, non-irradiated samples is low and shows only the slow nucleation regime similar to non-filtered irradiated samples (Figure 2, empty circles).

\begin{tabular}{|l|c|c|c|}
\hline Supersaturation & 1.4 & 1.5 & 1.6 \\
\hline Non-filtered irradiated & $21 \%$ & $60 \%$ & $94 \%$ \\
\hline Non-filtered non-irradiated & $2 \%$ & $11 \%$ & $10 \%$ \\
\hline Filtered irradiated & $3 \%$ & $12 \%$ & $30 \%$ \\
\hline Filtered non-irradiated & $1 \%$ & $3 \%$ & $24 \%$ \\
\hline
\end{tabular}

Table 1: Total percentage of samples crystallized at different supersaturations for each set of experiments. Total monitoring time was 5760 minutes (4 days). 


\section{Nucleation Kinetics:}

The cumulative probability distribution functions of induction times for non-filtered, irradiated samples are shown with a logarithmic time scale in Figure 3. The delay time required for the first crystal to be observed increases as the supersaturation is reduced. As described above, in the case of non-filtered irradiated samples, a bimodal nucleation regime is observed. This consists of an initial fast regime we identify as laser induced nucleation regime, and a second slower spontaneous nucleation regime. In order to describe the bimodal nucleation kinetics quantitatively, we have used a function based on a weighted sum of two exponential distributions with different time constants, as shown in Equation 2. This model assumes that there are two independent nucleation regimes, each with a constant nucleation rate.

$$
P(t)=A\left(1-\exp p^{\left(-\frac{t-t_{d}}{\tau_{1}}\right)}\right)+(1-A)\left(1-\exp \left(-\frac{t-t_{d}}{\tau_{2}}\right)\right) \quad \text { Eq. } 2
$$

Here $A$ is the fraction of samples that underwent laser induced nucleation, $\tau_{l}$ is the characteristic time for the laser induced nucleation regime, $\tau_{2}$ is the characteristic time for the spontaneous nucleation regime, $t_{d}$ is the delay time between laser irradiation and appearance of the first crystals and $t$ is time after laser irradiation. Note that the nucleation rate $J$ is related to the characteristic time of nucleation $\tau$ as $J V=1 / \tau$, where $V$ is the sample volume. ${ }^{27}$

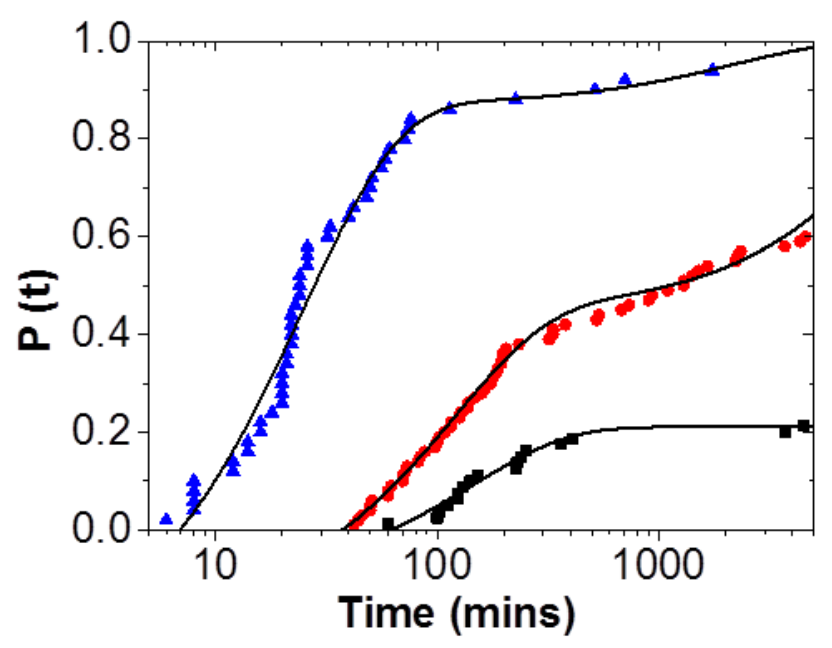


Figure 3: Fitting of biexponential model to cumulative probability distribution functions of induction times for supersaturations 1.4 (squares), 1.5 (circles) and 1.6 (triangles) for nonfiltered, irradiated samples.

Values for the proportion of samples that underwent laser induced nucleation (A), characteristic times for both laser-induced $\left(\tau_{1}\right)$ and spontaneous $\left(\tau_{2}\right)$ nucleation regimes, and the delay time $\left(t_{d}\right)$ representing the time required after nucleation for the incipient crystal to grow large enough to be detected by image analysis, were estimated by fitting a function represented by equation 1 to the experimental data by the method of non-linear least squares. Fitted delay times should correspond to growth times for crystals to reach about $1 \mathrm{~mm}$ at a given supersaturation and temperature. We estimated these times for both the $\alpha$ - and $\gamma$-polymorphs of glycine using previously published crystal growth rate data ${ }^{28}$. We found them to vary between approximately 10 and 25 minutes for the $\alpha$-polymorph and 30 to 80 minutes for the $\gamma$-polymorph over the range of supersaturations investigated, in a reasonable agreement with estimated delay time values.

In the fitting process, we calculate $95 \%$ confidence intervals for the estimated values based on the assumption that the experimental errors in evaluating each point in the cumulative distribution are Gaussian, uncorrelated and have a uniform standard deviation. Although it is difficult to be certain if these assumptions are strictly valid, the values nevertheless give an indication of the levels of uncertainty in the parameters extracted from the fits.

It can be seen from Table 2 that there is a strong increase in the proportion of samples that undergo laser-induced nucleation with increasing supersaturation: the value of A roughly doubles as supersaturation increases from 1.4 to 1.5 and doubles again from 1.5 to 1.6 . The characteristic time for laser induced nucleation regime $\left(\tau_{l}\right)$ decreases as supersaturation 
increases. There is also a decrease in the characteristic time for spontaneous nucleation regime $\left(\tau_{2}\right)$ with increasing supersaturation as expected, and these characteristic times are about two orders of magnitude longer than those for laser induced nucleation. This means that laser induced nucleation kinetics is about two orders of magnitude faster than spontaneous primary nucleation in this system.

\begin{tabular}{|c|c|l|l|l|}
\hline Supersaturation & $A$ & \multicolumn{1}{|c|}{$t_{d}(\mathrm{~min})$} & $\tau_{l}(\mathrm{~min})$ & \multicolumn{1}{c|}{$\tau_{2}(\mathrm{~min})$} \\
\hline 1.4 & $0.21( \pm 0.017)$ & $62.3 \pm 12.2$ & $143.4( \pm 36.6)$ & $1.48 \times 10^{6}\left(^{*}\right)$ \\
\hline 1.5 & $0.45( \pm 0.012)$ & $37.4 \pm 2.8$ & $117.0( \pm 8.6)$ & $1.14( \pm 0.16) \times 10^{4}$ \\
\hline 1.6 & $0.87( \pm 0.060)$ & $6.9 \pm 1.4$ & $24.7( \pm 4.0)$ & $2.17 \times 10^{3}\left(^{*}\right)$ \\
\hline
\end{tabular}

Table 2: Estimated values of parameters from fitting a biexponential model to cumulative probability distribution functions of induction times for non-filtered irradiated samples. $A$ is the proportion of samples that underwent laser induced nucleation, $t_{d}$ is the delay time between laser irradiation and appearance of the first crystals, $\tau_{1}$ is the characteristic time for laser induced nucleation, $\tau_{2}$ is the characteristic time for spontaneous nucleation. Quoted uncertainties represent $95 \%$ confidence intervals evaluated in the fitting process. Values marked with $\left(^{*}\right)$ are not statistically significant due to an insufficient number of data points.

In order to compare the characteristic time of the second nucleation regime, $\tau_{2}$, observed in non-filtered, laser irradiated samples, with the spontaneous nucleation rate observed in nonirradiated samples, we fitted a single-exponential function to the data for non-filtered nonirradiated samples. Due to an insufficient number of data points caused by slow nucleation, this was only done for samples with supersaturation 1.5. The value of the spontaneous nucleation characteristic time is shown as $\tau_{\text {spont }}$ in Table 3 and the fit to the data is shown in Figure 4. The 
characteristic time for spontaneous nucleation $\tau_{\text {spont }}$ for non-irradiated samples appears to be higher than the value of $\tau_{2}$ for the corresponding irradiated samples but the values are of a similar magnitude. To determine whether the cumulative probability distribution function of induction times for laser induced nucleation was consistent with a second regime governed by the rate of spontaneous nucleation, the bimodal fitting was repeated with $\tau_{2}$ fixed to be equal to $\tau_{\text {spont. }}$ The new fit to the data is shown in Figure 4 along with the original one. Although the new fit does not agree with the data quite as well, the results seem broadly consistent with a model in which the second nucleation regime has a rate that is equal to the rate of spontaneous nucleation. The values of the parameters, $A^{\prime}, \tau_{1}{ }^{\prime}$ and $t_{d}{ }^{\prime}$, resulting from the new fit are shown in Table 3 and can be seen to have similar values to the fit in which $\tau_{2}$ was varied.

\begin{tabular}{|c|c|c|c|c|}
\hline Supersaturation & $A^{\prime}$ & $t_{d}{ }^{\prime}(\mathrm{min})$ & $\tau_{l}{ }^{\prime}(\mathrm{min})$ & $\tau_{\text {spont }}(\mathrm{min})$ \\
\hline 1.5 & $0.49( \pm 0.011)$ & $34.0( \pm 5.0)$ & $145.7( \pm 12.9)$ & $3.43( \pm 0.26) \times 10^{4}$ \\
\hline
\end{tabular}

Table 3: Estimated values of parameters $A^{\prime}, t_{d}$ ' and $\tau_{1}$ ' from fitting a biexponential model to cumulative probability distribution function of induction times with a fixed value of $\tau_{2}=\tau_{\text {spont }}$ for non-filtered irradiated samples at supersaturation 1.5. 


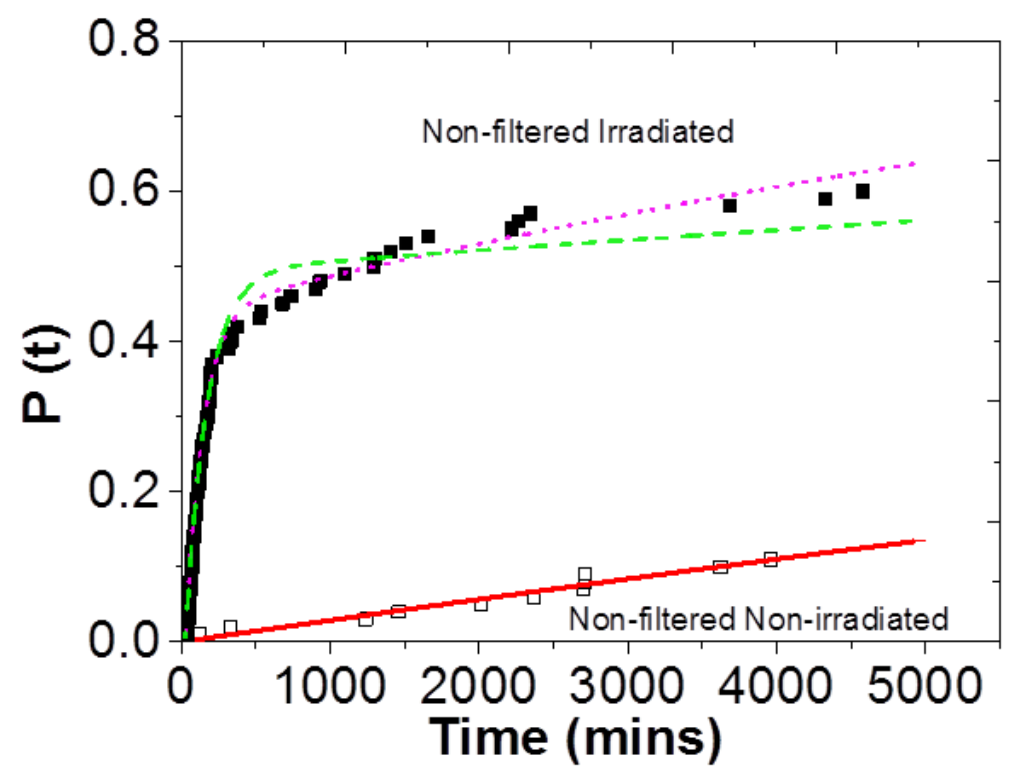

Figure 4: Cumulative probability distribution functions of induction times for irradiated and nonirradiated samples that had not been filtered, with supersaturation of 1.5. Best fits of single-exponential (non-irradiated samples) and biexponential (irradiated samples) models are shown. The singleexponential fit was used to estimate $\tau_{\text {spont }}$ for non-filtered non-irradiated samples (solid line). The biexponential fit has been performed both with $\tau_{2}$ fitted (dotted line) and fixed (dashed line) to the value of the spontaneous nucleation characteristic time $\tau_{\text {spont. }}$

\section{Effects of nanofiltration and laser irradiation on polymorphism}

Polymorphism analysis of the glycine crystals was performed using an ATR-FTIR spectrophotometer in the spectral range of $800 \mathrm{~cm}^{-1}$ to $1200 \mathrm{~cm}^{-1}$ at ambient temperature. Glycine is known to have three polymorphs at room temperature and atmospheric pressure, out of which the metastable $\alpha$-polymorph is commonly crystallised from aqueous solutions at nearneutral $\mathrm{pH} .{ }^{29}$ The $\alpha$-polymorph is kinetically stable at ambient conditions both in its dry form and in aqueous solutions at near neutral $\mathrm{pH}$. While the $\beta$-polymorph is only formed under 
specific conditions, ${ }^{30}$ the $\gamma$-polymorph is the thermodynamically stable form at ambient conditions and readily crystallises from aqueous solutions at high or low $\mathrm{pH}$ or in the presence of salts or other additives. ${ }^{31}$ The characteristic spectra of the $\alpha$ - and $\gamma$-polymorphs are shown in Figure 5A. The $\alpha$-polymorph exhibits a distinct absorption peak at $910 \mathrm{~cm}^{-1}$ and the $\gamma$-polymorph shows a characteristic peak at $927 \mathrm{~cm}^{-1} .32$ The polymorphic outcomes for filtered and nonfiltered samples, in the presence and absence of laser irradiation were determined this way and are discussed below.

In non-filtered irradiated samples differential polymorphism behaviour is observed. At low supersaturations (1.4) the $\alpha$-polymorph is strongly predominant and only one vial out of 14 which crystallized showed the $\gamma$-polymorph. By increasing the supersaturation to 1.5 and 1.6, the probability of formation of the $\gamma$-polymorph increases significantly to about $40 \%$ of the vials which crystallised. From a preliminary analysis on a limited number of samples it was noted that both the $\alpha$ - and $\gamma$-polymorph were formed in the fast laser induced regime, which indicates that nucleation of both polymorphs is enhanced by laser irradiation. However, crystals formed in the slow regime were almost exclusively the $\alpha$-polymorph, which is consistent with a spontaneous nucleation regime, as samples which were not irradiated similarly yielded almost exclusively the a-polymorph. In order to further confirm the solid form identification, single-crystal X-ray diffraction patterns were recorded for the crystals obtained from solutions at supersaturation 1.5 and we observed a similar percentage of the $\gamma$-polymorph as was determined by ATR-FTIR experiments. Clair et $a l^{26}$ have shown in a recent study that glycine solutions at $290 \mathrm{~K}$ irradiated with linearly polarized laser radiation with power density of $0.9 \mathrm{GW} / \mathrm{cm}^{2}$ produced $20-25 \%$ of the $\gamma$ polymorph for supersaturations of 1.4 and 1.6. These results quantitatively differ from the observations reported by Sun et al. ${ }^{12}$ where $100 \%$ of the $\alpha$-polymorph is observed for 
supersaturation 1.4 and $100 \%$ of the $\gamma$-polymorph is observed for supersaturations 1.5 and 1.6 for linearly polarized light.
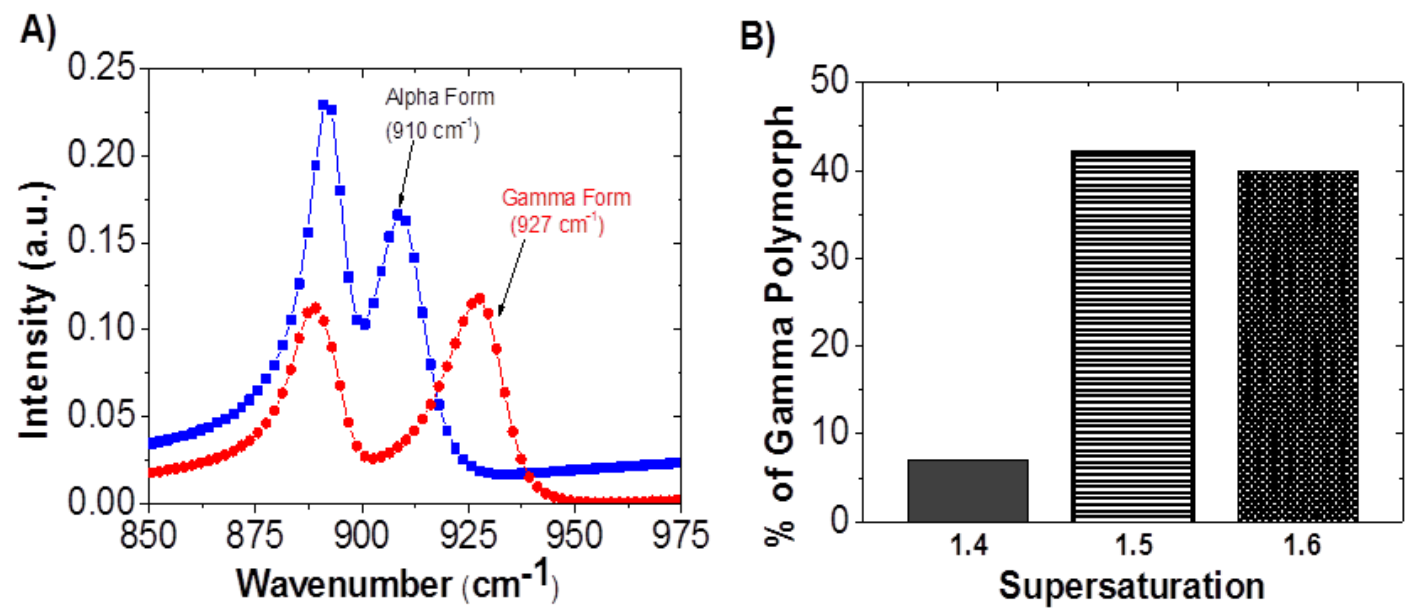

Figure 5: Polymorph characterization of glycine crystals obtained at various supersaturations by ATR-FTIR: A): Representative IR spectra for alpha and gamma polymorphs, B): Percentage of gamma polymorph obtained for non-filtered irradiated glycine solutions at various supersaturations.

Our results agree qualitatively with those of Sun et al. ${ }^{13}$ in terms of the variation in the polymorphic outcome in irradiated solutions, where at supersaturations of 1.5 and 1.6 they observed the $\gamma$-polymorph, while at supersaturation of 1.4 they observed the $\alpha$-polymorph. However we observed just $40 \%$ of the $\gamma$-polymorph for supersaturations 1.5 and 1.6 , in contrast to $100 \%$ observed by Sun et al. This could be due to intensity of laser pulses (our value is $0.4 \mathrm{GW} / \mathrm{cm}^{2}$ and they reported $0.7 \mathrm{GW} / \mathrm{cm}^{2}$ ). Our observations also differ from those of Clair et $a l{ }^{26}$, but some of these differences may be due to the fact that Clair et al. used a different irradiation geometry in which the laser beam exits the solution through the air-solution interface and the nucleation they observe occurs at that interface, while Sun et al. used a similar geometry to that used here, where the laser beams enters and exits the solution through glass walls and 
nucleation occurs in bulk solution. Due to the small number of nucleating samples for cases without laser irradiation or with filtration, there were too few results to give reliable information for numerical probabilities of polymorphic outcomes. Nevertheless, in all cases without laser irradiation or with filtration, the vast majority of crystals formed were $\alpha$-polymorphs at all supersaturations.

\section{Conclusions:}

We have demonstrated that laser induced nucleation in supersaturated glycine solutions is substantially suppressed by nanofiltration. An extensive set of experiments was used to construct cumulative probability distribution functions of induction times for irradiated and non-irradiated solutions with and without applying nanofiltration for various glycine supersaturations. The nucleation behaviour of irradiated non-filtered glycine solutions showed a bimodal distribution of induction times indicating two regimes; the first is a fast laser-induced nucleation regime and the second is a slow nucleation regime whose characteristic time is similar to that of spontaneous nucleation for non-irradiated samples. We estimated nucleation rates in the laser-induced nucleation regime and found them to be about two orders of magnitude higher than those in the spontaneous nucleation regime. These results suggest that filtration can remove some entities in the solutions whose interactions with the laser radiation are critical to induce nucleation. This conclusion is inconsistent with previously proposed theories of laser induced nucleation being due to molecular-scale interactions involving solute molecules and solvent alone since such interactions should not be affected by the nanofiltration process. Differential polymorphism behaviour was observed for different supersaturations. Solutions at a lower supersaturation (1.4) almost exclusively yielded the $\alpha$-polymorph under all conditions. The non-filtered samples at higher supersaturations (1.5 and 1.6) showed a significant presence of the $\gamma$-polymorph (40\%) 
when irradiated, while non-irradiated solutions nucleate almost exclusively the $\alpha$-polymorph at all supersaturations. Nanofiltration of glycine solutions across all supersaturations results in the $\alpha$-polymorph irrespective of whether the solutions are irradiated or not. These results indicate that colloidal-scale solute structures, external particulates or molecular impurities that can be removed by nanofiltration play a major role in laser-induced nucleation and polymorphism control. Proposed mechanisms for laser induced nucleation in this system will need to take into account interactions of laser light with such solute structures or impurities.

AUTHOR INFORMATION

Corresponding Author

*Email: Jan.Sefcik@strath.ac.uk

Author Contributions

$\$$ These authors contributed equally. (NJ and TK)

\section{Supporting information}

Schematic and image of the sample monitoring set up is available online free of charge as supporting information file.

\section{Funding Sources}

EPSRC Manufacturing with Light (EP/L022281/1), EPSRC CIM (EP/I033459/1), CMAC DTC (EP/K503289/1).

\section{ACKNOWLEDGMENT}


We acknowledge the support of the Engineering and Physical Sciences Research Council

(EPSRC) for funding through Manufacturing with Light (EP/L022281/1), CIM (EP/I033459/1) and DTC (EP/K503289/1).

\section{ABBREVIATIONS}

PES, Polyethersulfone; NPLIN, Non-photochemical laser induced nucleation; LIN, Laser induced nucleation.

\section{For Table of Contents Use Only}

Manuscript Title

Filtration suppresses laser induced nucleation of glycine in aqueous solutions

Author List

Nadeem Javid, Thomas Kendall, Iain S. Burns and Jan Sefcik

TOC graphic

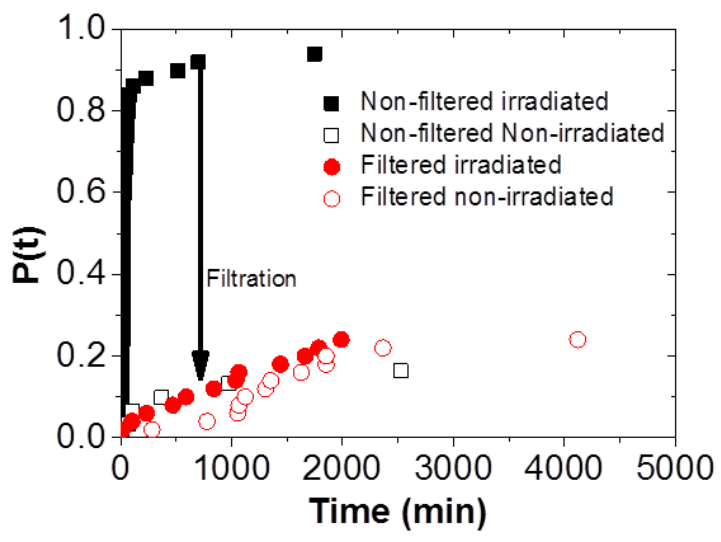

Synopsis 
Nanofiltration $(0.2 \mu \mathrm{m}$ polyethersulfone filter membrane) of aqueous glycine solutions has a

pronounced effect in suppressing laser-induced nucleation.

${ }^{1}$ Baillie, T. A. Metabolism and Toxicity of Drugs. Two Decades of Progress in Industrial Drug Metabolism, Chem.
Res. Toxicol., 2008, 21, 129-137
${ }^{2}$ Rodríguez-Spong, B.; Price, C. P.; Jayasankar, A.; Adam J. Matzger, A. J.; Rodríguez-Hornedo, N. General
principles of pharmaceutical solid polymorphism: a supramolecular perspective, Adv Drug Deliver Rev 2004 56,
$241-274$
${ }^{3}$ Morissette, S. L.; Almarsson, Ö.; Peterson, M. L.; Remenar, J. F.; Read, M. J.; Lemmo, A. V.; Ellis, S.; Cim, M. J.; Gardne, C. R.; High-throughput crystallization: polymorphs, salts, co-crystals and solvates of pharmaceutical solids, Adv Drug Deliver Rev 2004, 56, 275-300

${ }^{4}$ Zhou, G. X.; Fujiwara, M.; Woo, X. Y.; Rusli, E.; Tung, H.; Starbuck, C.; Davidson, O.; Ge, Z.; Braatz. R. D. Direct Design of Pharmaceutical Antisolvent Crystallization through Concentration Control, Crystal Growth \& Design, 2006 , 4, 892-898

5 Muller, F. L.; Fielding, M.; Black, S. A Practical Approach for Using Solubility to Design Cooling Crystallisations, Org. Process Res. Dev. 2009, 6, 1315-1321.

${ }^{6}$ Zamaninpoor, M. H.; Mancera, R. L.; The emerging application of ultrasound in lactose crystallisation, Trends Food Sci Tech, 2014, 38, 47-59

${ }^{7}$ Forsyth, C.; Mulheran, P. A.; Forsyth, C.; Haw, M. D.; Burns, I. S.; Sefcik, J.; Influence of Controlled Fluid Shear on Nucleation Rates in Glycine Aqueous Solutions. Cryst. Growth Des. 2015, 15, 94-102

${ }^{8}$ Koizumi, H.; Uda, S.; Fujiwara, K.; Tachibana, M.; Kojima, K.; Nozawa, J.; Crystallization of high-quality protein crystals using an external electric field. J.Appl.Cryst. 2015. 48, 1507-1513

9 Garetz, B. A.; Aber, J. E.; Goddard, N. L.; Young, R. G.; Myerson, A. S. Nonphotochemical, PolarizationDependent, Laser-Induced Nucleation in Supersaturated Aqueous Urea Solutions. Phys. Rev. Lett, 1996 77, $3475-$ 3476

${ }^{10}$ Duffus, C.; Camp, P. J.; Alexander, A. J.; Spatial Control of Crystal Nucleation in Agarose Gel, J. AM. Chem. Soc. 2009, 131, 11676-11677

11 Ikni, A.; Clair, B.; Scouflaire, P.; Veesler, S.; Gillet, J.; Hassan, N. E.; Dumas, F.; Bire, A. S.; Experimental Demonstration of the Carbamazepine Crystallization from Non-photochemical Laser-Induced Nucleation in Acetonitrile and Methanol, Cryst. Growth Des, 2014, 14, 3286-3299

12 Garetz, B. A.; Matic, J.; Myerson. A. S.; Polarization Switching of Crystal Structure in the Nonphotochemical Light-Induced Nucleation of Supersaturated Aqueous Glycine Solutions, Phys. Rev. Lett, 2002, 89, 175501

${ }^{13}$ Sun, X.; Garetz, B. A.; Myerson. A. S.; Supersaturation and Polarization Dependence of Polymorph Control in the Nonphotochemical Laser-Induced Nucleation (NPLIN) of Aqueous Glycine Solutions, Cryst Growth Des, 2006, 6, 684-689

${ }_{14}$ Zaccaro, J.; Matic, J.; Myerson, A. S.; Garetz, B. A.; Nonphotochemical, Laser-Induced Nucleation of Supersaturated Aqueous Glycine Produces Unexpected $\gamma$-Polymorph, Cryst Growth Des, 2001, 1, 5-8

${ }_{15}$ Yuyama, K.; Rungsimanon, T.; Sugiyama, T.; Masuhara, H. Selective Fabrication of $\alpha$ - and $\gamma$-Polymorphs of Glycine by Intense Polarized Continuous Wave Laser Beams, Cryst. Growth Des. 2012, 12, 2427-2434

${ }^{16}$ Alexander, A. J.; Camp, P. J. Single Pulse, Single Crystal Laser-Induced Nucleation of Potassium Chloride, Cryst Growth Des, 2009, 9, 958-963.

${ }^{17}$ Ward, M. R.; Jamieson, W. J.; Leckey, C. A.; Alexandera, A. J. Laser-induced nucleation of carbon dioxide bubbles, J. Chem. Phys. 2015, 142, 144501

${ }^{18}$ Soare. A.; Dijkink. R.; Pascual. M. R.; Sun. C.; Cains. P. W.; Lohse, D.; Stankiewicz, A. I.; Kramer, H. J. M.; Crystal Nucleation by Laser-Induced Cavitation, Cryst Growth Des. 2011, 11, 2311-2316

${ }^{19}$ B. C. Knott, B. C.; LaRue, J. L.; Wodtke, A. M.; Doherty, M. F.; Peters, B. Bubbles, crystals, and laser-induced nucleation, J. Chem. Phys, 2011,134, 171102

${ }^{20}$ Ward, M.; Alexander, A. J. Nonphotochemical Laser-Induced Nucleation of Potassium Halides: Effects of Wavelength and Temperature, Cryst. Growth Des. 2012, 12, 4554-4561

${ }^{21}$ Nakamura. K.; Hosokawa, Y.; Masuhara, H. Anthracene Crystallization Induced by Single-Shot Femtosecond Laser Irradiation: Experimental Evidence for the Important Role of Bubbles, Cryst Growth Des, 2007, 7, 885-889. 
${ }^{22}$ Jawor-Baczynska, A.; Sefcik, J.; Moore B. D.; $250 \mathrm{~nm}$ Glycine-Rich Nanodroplets Are Formed on Dissolution of Glycine Crystals But Are Too Small To Provide Productive Nucleation Sites Cryst. Growth Des. 2013, 13, 470-478

23 Jawor-Baczynska. A, Moore. B. D, Lee. H. S, McCormick. A. V, Sefcik. J, Population and size distribution of solute-rich mesospecies within mesostructured aqueous amino acid solutions, Faraday Discuss., 2013, 167, 425-440

${ }^{24}$ Lee. I. S.; Evans. J. M. B.; Erdemir. D.; Lee. A. Y.; Garetz. B. A.; Myerson. A. S.; Nonphotochemical Laser Induced Nucleation of Hen Egg White Lysozyme Crystals, Crystal Growth \& Design, 2008, 12, 4255-4261

${ }^{25}$ Yi. Y.; Hatziavramidis. D,; Myerson. A. S,; Waldo. M,; Beylin. V. G,; Mustakis. J,; Development of a SmallScale Automated Solubility Measurement Apparatus, Ind. Eng. Chem. Res. 2005, 44, 5427-5433

${ }^{26}$ Clair, B.; Ikni, A.; Li, W.; Scouflaire, P.; Quemenerd, V.; Bire, A. S. A new experimental setup for highthroughput controlled non-photochemical laser-induced nucleation: application to glycine crystallization. J. Appl. Cryst. 2014. 47, 1252-1260

27 Jiang, S.; ter Horst, J. H. Crystal Nucleation Rates from Probability Distributions of Induction Times, Cryst. Growth Des, 2011, 11, 256-261

${ }^{28}$ Dowling, R.; Davey, R. J.; Curtis, R. A.; Han, G. J.; Poornachary, S. K.; Chow, P. S.; Tan, R. B. H. Acceleration of crystal growth rates: an unexpected effect of tailor-made additives. Chem. Commun. 2010, 46, 5924-5926

${ }^{29} \mathrm{Yu}, \mathrm{L}$; $\mathrm{Ng}, \mathrm{K}$; Glycine Crystallization during spray drying: The $\mathrm{pH}$ effect on salt and polymorphic forms, $J$. Pharm. Sci. 2002, 91, 2367-2375

${ }^{30}$ Hamilton, B. D.; Hillmyer, M. A.; Ward, M. D.; Glycine Polymorphism in Nanoscale Crystallization Chambers Cryst. Growth Des. 2009, 8, 3368-3375

${ }^{31}$ Moolya, B. N., Jayarama, A., Sureshkumar, M. R., Dharmaprakash, S. M. Hydrogen bonded nonlinear optical $\gamma-$ glycine: Crystal growth and characterization. J. Cryst. Growth. 2005, 280, 581-586.

32 Yang, X.; Lu, J.; Wang, X.; Ching, C. Effect of sodium chloride on the nucleation and polymorphic transformation of glycine. J. Cryst. Growth, 2008, 310, 604-611 